\title{
Accompagner la transformation numérique des PME : une perspective écosystémique de la création de valeur
}

\author{
Claudia Pelletier ${ }^{\mathrm{a}}$, Vanessa Martel ${ }^{\mathrm{b}}$
}

RÉSUMÉ. Dans le monde actuel des affaires, la transformation numérique des PME dépasse désormais le simple choix d'un « outil » technologique. Cela suppose aussi des mutations profondes dans l'organisation, incluant le capital social. Une perspective écosystémique de ce phénomène permet de mieux comprendre comment les acteurs en présence intègrent de nouvelles ressources, tangibles et intangibles, par l'échange de services avec des intervenants socioéconomiques et des spécialistes en technologies de l'information (TI). En organisant la transformation numérique dans un système soutenant la cocréation de valeur, on observe ainsi l'émergence d'une panoplie de programmes visant l'accompagnement de cette transformation. Or, qu'en estil vraiment de ces échanges entre acteurs indépendants? De quoi sont-ils constitués plus précisément? L'exploration des pratiques d'accompagnement au numérique existantes met de l'avant une logique de services qui se combine aux principes reconnus de l'innovation en contexte de PME. Des contributions théoriques et pratiques complètent le portrait présenté.

ABSTRACT. In the actual business context, the digital transformation of SMEs goes beyond the simple choice of a technological "tool". This situation also supposes profound changes in the organization, including the social capital. An ecosystemic perspective of this phenomenon provides a better understanding of how the actors integrate new tangible and intangible resources, through the exchange of services between socioeconomic professionals and information technology (IT) specialists. Organizing digital transformation in a system that supports cocreation of value allows to observe the emergence of an array of programs intended to accompany this transformation. But what is the actual situation with these exchanges between independent actors? How are they constituted more specifically? Exploring the digital transformation support practices reveals a logic of services that combines with recognized principles of innovation in the SMEs context. Theoretical and practical contributions complete the observed situation.

\section{Introduction}

En 2017, les microentreprises, c'est-à-dire les entreprises qui comptent de 1 à 4 employés, représentaient $53,8 \%$ des entreprises canadiennes. Si on y ajoute celles qui comptent entre 5 et 9 employés, ce pourcentage atteint $73,4 \%$. Cela signifie que près de 3 entreprises sur 4 au Canada comptaient entre 1 et 9 employés (Innovation, Sciences et Développement économique Canada, 2019). La transformation numérique de ces entreprises, dont l'importance s'accroît autant qu'elle se complexifie (Weiß, Zolnowski, Warg et Schuster, 2018), souligne le besoin de consolider ou de développer les compétences appropriées sur différents plans, technologique certes, mais aussi managérial et relationnel (OCDE, 2016; Prasad et Green, 2016).

Pour ce faire, certains organismes de soutien dédiés aux petites et moyennes entreprises (PME) offrent des ressources qui incluent différentes formes d'accompagnement plus ou moins formalisées (Gregory, Keil, Muntermann et Mähring, 2015). Ces mesures sont aussi généralement cohérentes avec une définition large de la notion de formation de même qu'avec celle d'accompagnement (Barthelémy et Le Pennec, 2018) puisque l’idée est alors d'offrir le «moyen de donner du sens, une

\footnotetext{
a Professeure en systèmes d'information, DBA, membre de l'Institut de recherche sur les PME INRPME, Université du Québec à Trois-Rivières

${ }^{\mathrm{b}}$ Entrepreneure et consultante, MBA en gestion de l'innovation dans les PME, Université du Québec à Trois-Rivières
} 
direction ou une raison d'être à quelque chose » (p. 9), notamment par l'intermédiaire d'une « interaction avec l'autre » (p. 186). Il est donc intéressant de voir comment cette notion d'accompagnement peut s'appliquer au domaine des technologies de l'information (TI) et aux compétences dites numériques dans le contexte des affaires d'une PME.

Pour le monde des affaires et les PME qui vivent cette transformation à travers leurs processus, leurs structures autant que les relations avec leur environnement (Dyer et Kale, 2007), les écosystèmes d'affaires (Tsujimoto, Kajikawa, Tomita et Matsumoto, 2018), les réseaux (Daidj, 2011) de même que les ressources matérielles et humaines pour l'innovation qui s'y trouvent revêtent alors une importance de premier ordre (de Vasconcelos Gomes, Facin, Salerno et Ikenami, 2018; Zollo, Bettinazzi, Neumann et Snoeren, 2016). Fondée sur l'interdépendance des acteurs plutôt que sur le simple échange de ressources entre eux (Barney, Ketchen et Wright, 2011), cette importance se manifeste entre autres par l'offre grandissante de programmes de formation et d'accompagnement au numérique. Ces programmes de toutes sortes conçus et offerts par des intervenants socioéconomiques, par des spécialistes des TI et par d'autres consultants vantent (et vendent, dans certains cas) l'adaptation à un monde de plus en plus numérique de différentes manières (Gregory et collab., 2015; Pelletier et Martel, 2019).

Cette situation fait également en sorte que les partenariats avec d'autres organisations et les services offerts par divers organismes s'avèrent désormais non seulement essentiels pour plusieurs PME (OCDE, 2016; Raymond et Uwizeyemungu, 2017), mais également critiques pour leur survie, en particulier les plus petites d'entre elles (Pelletier et Cloutier, 2019). Or, qu'en est-il de ces échanges avec des acteurs extérieurs à l'entreprise? De quoi sont-ils constitués plus précisément? Et, surtout, comment ces échanges contribuent-ils à une création de valeur fondée sur les TI (IT value creation) pour les PME et les acteurs qui s'y trouvent?

La présente démarche s'appuie sur une étude préalable ayant recensé et analysé 19 types d'accompagnement au numérique. Toutes destinées à encourager le virage numérique auprès des entrepreneurs et PME du Québec, les pratiques alors identifiées allaient des cellules d'expertise au coaching, en passant par les incubateurs/accélérateurs, les fablabs ainsi que les différents programmes offerts par les gouvernements provincial et municipal (Pelletier et Martel, 2019).

L'article présenté ici discute des mécanismes qui (re)définissent les comportements, de même que des relations entre les acteurs de la transformation numérique dans ce contexte (entrepreneurs, dirigeants, consultants spécialisés, intervenants socioéconomiques, organismes de soutien, bailleurs de fonds, etc.). Plus précisément, ces mécanismes sont explorés à partir des questions de recherche suivantes : Comment se réalise l'accompagnement à la transformation numérique dans un écosystème d'affaires dédié aux entrepreneurs et PME? Qu'estce qui caractérise les relations qu'entretiennent alors les différents acteurs de cette transformation numérique?

Exploratoire et inspirée des principes de la recherche évaluative (Kushner, 2017), la démarche s'appuie sur un design de recherche qualitatif où se côtoient des méthodes de collecte et des sources diversifiées de données adaptées au contexte social du phénomène étudié (Gross, 2009; Maxwell, 2012). Suggérant une situation plus disparate que celle qu'on aurait pu anticiper a priori, les résultats révèlent une panoplie de pratiques d'accompagnement au numérique qui poursuivent des objectifs louables certes, mais qui soulèvent néanmoins des questions importantes quant aux mécanismes d'échange qui sont à l'œuvre (Dyer et Kale, 2007; Zollo et collab., 2016), de même qu'à leurs véritables retombées pour les entreprises et les dirigeants qui en bénéficient. Ce faisant, on verra aussi poindre la complexité des besoins auxquels on tente de répondre sur le plan des compétences TI (aussi dites «numériques») recherchées par les acteurs à travers l'accompagnement en question (Gregory et collab., 2015).

Pour ce faire, l'article présente d'abord le contexte et les fondements de la recherche, une brève description de la méthodologie, puis les résultats et une discussion. Le tout est complété des contributions théoriques et pratiques, incluant différents apprentissages qu'on peut en tirer et qui sont présentés sous forme de recommandations. 


\section{Contexte et fondements}

Le présent article adopte une perspective écosystémique de la transformation numérique des PME. La collaboration externe représente ainsi un des moteurs de la création de valeur fondée sur les TI (IT value creation) (Prasad et Green, 2016), et ce, dans un contexte dit social qui dépasse les frontières habituelles de l'entreprise (Dyer et Kale, 2007; Gross, 2009). Cette perspective nous suggère des pistes quant aux liens à faire avec l'écosystème immédiat de l'entreprise (Tsujimoto et collab., 2018), avec ses réseaux (Daidj, 2011) et avec le recours à la cocréation de valeur fondée sur les TI à des fins d'innovation (de Vasconcelos Gomes et collab., 2018).

Dans le contexte de la recherche, cette cocréation (value cocreation) est fondée sur une logique dominante de services (service-dominant logic ou SDL) qui s'installe entre des acteurs diversifiés et représentant généralement des entités indépendantes les unes des autres (Lusch et Nambisan, 2015; Ng et Vargo, 2018). C'est la raison pour laquelle nous adoptons la définition proposée par Laudon, Laudon et Costa (2017) concernant ce phénomène de l'ère numérique :

La transformation numérique est un processus qui consiste à réaliser ou à faciliter presque toutes les relations d'affaires significatives avec les clients, les fournisseurs et les employés à l'aide des technologies de l'information (TI). Les activités et les processus liés à l'activité essentielle de l'entreprise s'établissent alors à l'aide de réseaux numériques présents dans toute l'entreprise ou reliant plusieurs entreprises entre elles (p. 13).

Dans ce sens, on comprend que la transformation numérique est réalisée entre des acteurs dont les besoins et les contributions respectifs reposent désormais sur l'interdépendance, plutôt que sur des ressources et des actifs simplement « consommés » par l'entreprise (Barney et collab., 2011) ou " échangés » entre un client et son fournisseur (de Vasconcelos Gomes et collab., 2018). Ce faisant, la création de valeur fondée sur les TI deviendra une "cocréation» (Vargo et Lusch, 2017), laquelle concerne plus précisément les TI et les compétences numériques et qui repose désormais davantage sur une logique et des systèmes destinés à soutenir la transformation numérique des PME. En d'autres mots, il s'agit de systèmes dits "sociaux", où se côtoient et interagissent différents éléments de l'organisation et de son environnement afin de servir l'innovation au sens large (Trépanier, St-Pierre et Bertrand, 2012; Vargo et Akaka, 2012).

Dépassant l'idée du simple « outil» technologique à acquérir (Neirotti, Raguseo et Paolucci, 2018; OCDE, 2016), cette logique complexe de l'acquisition et du développement des compétences numériques s'avère également conforme aux défis qui se posent dans le contexte actuel du monde des affaires et de l'adaptation organisationnelle, quelle que soit sa nature (Weiß et collab., 2018; Zollo et collab., 2016). Cela inclut tout ce qu'une telle transformation, interne et externe, peut entrainer pour les PME et leurs dirigeants, et ce, autant sur les plans technologique, managérial que relationnel (Laudon et collab., 2017; Raymond et Uwizeyemungu, 2017).

\subsection{Accompagner la transformation numérique des PME : plus qu'un simple outil à acquérir}

De nos jours, la transformation numérique sousentend une étendue qui dépasse le système informatisé développé ou vendu par un fournisseur spécialisé (Neirotti et collab., 2018). Par la même occasion, on constate l'émergence de besoins variés autant dans l'organisation elle-même que dans son écosystème, où seront vraisemblablement recherchés des services plus ou moins spécialisés, ainsi que des programmes de soutien et d'accompagnement au numérique (Gregory et collab., 2015; Weiß et collab., 2018).

Ainsi, certains organismes de soutien dédiés aux PME offriront différentes formes d'accompagnement plus ou moins formalisées. Ces ressources seront généralement cohérentes avec une définition large de la notion de formation en tant que « moyen de délivrer un contenu» (Barthelémy et Le Pennec, 2018, p. 186). Quant à l'accompagnement dit professionnel, il est défini en tant que «moyen de donner du sens, une direction ou une raison d'être à quelque chose » (p. 9), et ce, par l'intermédiaire d'une «interaction avec l'autre afin de l'aider à faire émerger ses propres idées » (p. 186). Dans le cadre de la présente recherche, il est donc intéressant de constater comment ces notions de formation et d'accompagnement peuvent s'appliquer à l'adoption et à l'utilisation des TI, ainsi qu'aux compétences dites numériques. 
Pour les PME en particulier, la transformation numérique exige désormais la planification et la réalisation d'une véritable stratégie numérique qui dépasse l'acquisition de la dernière application à la mode (Pelletier et Cloutier, 2019). Basée sur des pratiques, sur des stratégies et sur des relations nouvelles avec l'environnement externe (Neirotti et collab., 2018), une approche de création de valeur fondée sur les TI souligne que les défis de cette transformation sont plus que jamais technologiques, managériaux et organisationnels (Laudon et collab., 2017). En outre, cette création de valeur sous-entend des échanges et des contributions qui varient au gré des compétences individuelles et des capacités organisationnelles des acteurs en présence. Acteurs qui sont non seulement en interactions constantes (Prasad et Green, 2016), mais aussi de plus en plus complémentaires (Zollo et collab., 2016). Utilisés pour qualifier les impacts possibles des types d'accompagnement au numérique recensés lors de l'étude sur laquelle s'appuie la présente démarche (Pelletier et Martel, 2019), différents éléments du modèle INNOSTICMD1 suggèrent de manière similaire que la transformation numérique est constituée d'éléments tangibles et intangibles tels que les relations, les pratiques d'affaires, les modes de travail et les ressources (Trépanier et collab., 2012).

\section{2 Écosystèmes et réseaux en soutien à la transformation numérique des PME}

Les définitions du concept d'écosystème sont nombreuses dans les écrits scientifiques, mais réfèrent généralement à diverses formes d'organisations en réseau qui sont parfois territoriales, parfois non (Daidj, 2011). Selon Tsujimoto et ses collègues (2018), les définitions du concept d'écosystème varient également selon les différentes perspectives qui sont adoptées, soit : 1) l'écologie industrielle destinée à un secteur en particulier; 2) l'écosystème ou l'environnement dédié aux affaires en général; 3) le développement et l'usage d'une plateforme de gestion autour de laquelle s'organisent les échanges; et, enfin, 4) la participation à des réseaux multiacteurs où se combinent des éléments des trois autres perspectives concernant les activités réalisées, de même que la compétition pour l'obtention de ressources et la coopération pour leur mise en valeur. Ces mêmes auteurs indiquent que ces perspectives se distinguent toutefois par les attributs des acteurs (p. ex., organismes publics, entreprises privées, utilisateurs et destinataires, etc.) ainsi que par ce qui est au cœur des échanges réalisés (p. ex., ressources matérielles, savoirs et connaissances, financement, ententes contractuelles, services de nature commerciale ou institutionnelle, etc.).

Selon Daidj (2011), on peut constater rapidement que la notion d'écosystème recoupe plusieurs éléments de la notion de réseaux. Premièrement, parce qu'elle adopte également différentes perspectives (p. ex., réseaux industriels dédiés soit à la croissance, soit à l'innovation; regroupements géographiques, aussi nommés clusters ou réseaux interentreprises, et où domine notamment l'interaction entre entreprises distinctes et indépendantes). Deuxièmement, parce qu'elle suggère différentes formes d'organisations autour d'éléments qui seront soit formels (p. ex., ententes contractuelles, besoins économiques comblés par des institutions, etc.), soit informels (p. ex., relations de complémentarité, échanges de compétences, innovation ouverte, collaborations variées, etc.). En résumé, la notion d'écosystème appliquée au monde des affaires est en fait « une forme d'organisation en réseau » qui s'apparente à un «réseau d'innovation non territorialisé » (Daidj, 2011, p. 125), d'où le constat qu'on trouve plus d'éléments communs à ces deux notions que d'éléments de réelle distinction.

Cela dit, les éléments qui précèdent concernant les échanges et les interactions entre entités distinctes s'avèrent similaires à ce qui se vit actuellement dans le domaine de la transformation numérique et de ses différents modes d'accompagnement (Pelletier et Cloutier, 2019; Pelletier et Martel, 2019). Dans ces conditions, on pourra donc voir évoluer l'écosystème dédié aux affaires vers un écosystème d'innovation dédié à la transformation numérique, qui sera plus révélateur des véritables activités qui s'y réalisent (de Vasconcelos Gomes et collab., 2018). Ce qui pourra alors varier concerne, par exemple, le degré de proximité des acteurs, l'intensité et la nature de leurs relations ou encore le degré et l'ouverture de leurs contributions respectives à une plateforme numérique commune, dans certains cas (Daidj, 2011; Tsujimoto et collab., 2018).

En matière de transformation numérique, les systèmes et les réseaux intraorganisationnels et extraorganisationnels qui se forment regroupent 
désormais « des entrepreneurs et des investisseurs privés, des innovateurs extérieurs aux pipelines traditionnels de l'entreprise, des utilisateurs/communautés d'utilisateurs, des fonctionnaires/décideurs gouvernementaux et des consortiums » (Tsujimoto et collab., 2018, p. 6). Devant une transformation de cet ordre, on peut alors anticiper l'émergence d'un écosystème où la complexité, le rythme autant que la diversité des échanges vont s'accroittre, notamment à travers différents réseaux interconnectés, qu'ils soient territorialisés ou non (Daidj, 2011).

\subsection{Logique de services pour la transformation numérique des PME}

Suivant ce qui précède, l'élément-clé de la transformation numérique des PME sera la recherche d'innovations qui peuvent se réaliser en plusieurs temps et dans plusieurs lieux de l'entreprise (Trépanier et collab., 2012). D'abord et tel que suggéré par de Vasconcelos Gomes et ses collègues (2018), lors de la création de valeur elle-même (p. ex., l'adoption et l'utilisation généralisée d'une nouvelle application numérique qui donne lieu à l'optimisation d'une pratique dont bénéficie l'ensemble de l'entreprise). Deuxièmement par la capture de cette valeur dans l'entreprise ou, encore mieux, dans l'écosystème plus large (p. ex., lorsque la nouvelle application numérique utilisée donne lieu à une transformation en profondeur d'une pratique, d'un produit ou d'un service par plusieurs acteurs, à la fois internes et externes). Deux activités distinctes, quoique complémentaires, mais dont le point commun est de dépasser le comportement des individus pour mettre de l'avant celui des organisations et des systèmes sociaux dans lesquels elles évoluent (Prasad et Green, 2016).

Dans ce contexte, les acteurs d'un écosystème donné sont notamment appelés à explorer l'intégration des ressources, les contraintes et les leviers des échanges qui se produisent, les arrangements de nature institutionnelle, ainsi que la nature des services échangés et leurs interrelations (Vargo et Lusch, 2017). Ils adoptent alors une logique dominante de services (SDL), où la base de tous les échanges consiste en l'application des compétences au bénéfice d'une autre entité que sa propre organisation (Vargo et Akaka, 2012). À cet égard, il importe toutefois de mentionner qu'une telle logique n'exclut pas la présence d'acteurs dont les activités seront rémunérées (Gregory et collab., 2015). Par exemple, lorsqu'une agence spécialisée offre ses services pour le développement d'un site web ou la gestion des médias sociaux, ou encore lorsqu'une intervention d'accompagnement faite par un consultant externe est subventionnée, partiellement ou entièrement, par une mesure ou un programme gouvernemental destiné à encourager le virage numérique des $\mathrm{PME}^{2}$.

Issue des travaux de Vargo, Lusch et leurs collègues depuis le milieu des années 2000, la figure 1 présente l'adaptation du cadre conceptuel de cette logique. Un cadre qui s'avère cohérent avec les notions d'écosystèmes, d'affaires ou d'innovation ainsi que de réseaux (Daidj, 2011; de Vasconcelos Gomes et collab., 2018; Tsujimoto et collab., 2018).

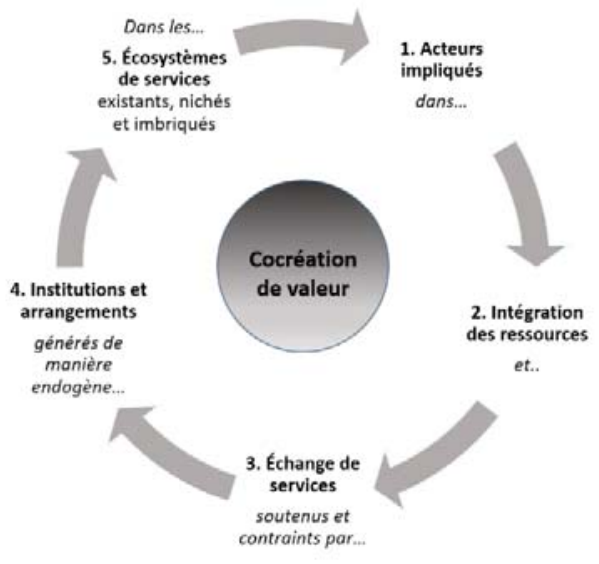

Figure 1 - Cadre conceptuel de la logique dominante de services (SDL) Source : Adaptation de Vargo et Lusch, 2017, p. 49 
Dans ce cadre permettant le développement d'une perspective écosystémique de la transformation numérique des PME, l'accent est mis sur les processus permettant la création de valeur, plutôt que sur les actifs qui y sont acquis (Vargo et Akaka, 2012). De plus, également conforme aux défis qui se posent dans le contexte de la transformation des entreprises à l'ère numérique (Pelletier et Cloutier, 2019; Weiß et collab., 2018), les différents acteurs en présence misent sur des relations qui se (re)définissent entre les entrepreneurs, les dirigeants de PME, les consultants et autres spécialistes des TI, ainsi que les intervenants socioéconomiques et institutionnels en présence (Gregory et collab., 2015; OCDE, 2016).

Cette approche met alors en évidence le dynamisme et la complexité des réseaux composés d'acteurs provenant d'organisations distinctes et autonomes (Daidj, 2011). Réseaux soutenant, par la même occasion, l'émergence, l'approfondissement et le maintien de relations d'affaires fondées sur l'acquisition et sur le développement de compétences numériques adaptées aux PME, surtout si ces relations visent le développement de capacités nouvelles entourant une innovation ainsi que le partage de connaissances, de technologies, d'habiletés et de ressources (de Vasconcelos Gomes et collab., 2018). Cela, tel qu'il est de plus en plus fréquent de le voir grâce aux différents types d'accompagnement au numérique offerts dans les écosystèmes dédiés à la transformation numérique des PME (Pelletier et Martel, 2019).

\section{Méthodologie}

Dans le cadre de la démarche d'observation réalisée au préalable auprès des organismes et des intervenants socioéconomiques, le projet a eu recours à un design de recherche qualitatif (Maxwell, 2012). L'approche adoptée a été structurée selon les principes de la recherche évaluative (Kushner, 2017), dont les grandes étapes sont présentées à la figure 2 .

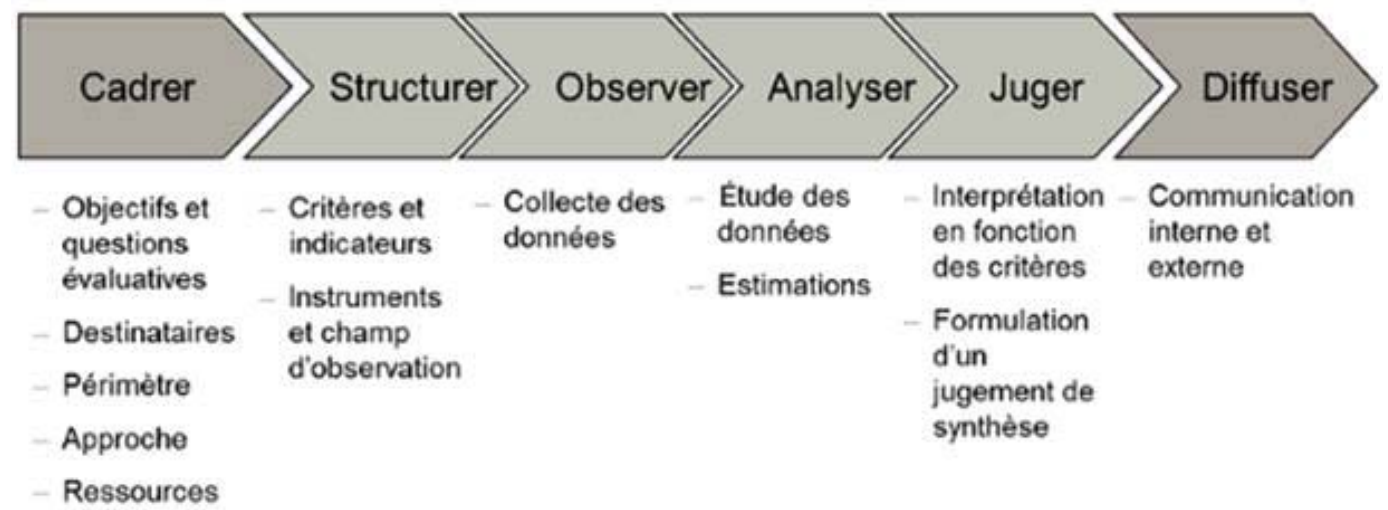

Figure 2-Étapes d'une recherche évaluative menée en contexte social Source : Tiré de Sibieude et Claverie, 2011, p. 18

\section{1 Étape 1 : Cadrer}

Une recension sommaire de la littérature a été faite, notamment concernant l'état des connaissances actuelles ainsi que les définitions et catégorisations de compétences numériques dont il pouvait être question. À cette étape, les membres de l'équipe de recherche ont aussi identifié les partenaires et les collaborateurs potentiels, de même qu'établi le calendrier et l'échéancier afin d'atteindre les objectifs.

\section{2 Étape 2 : Structurer}

Ici, les outils et les méthodes nécessaires au processus de recherche ont été sélectionnés. En raison de la dimension sociale de la recherche (Gross, 2009), une approche qualitative par triangulation des sources et des méthodes de collecte a été privilégiée (Maxwell, 2012). Elle comportait des recherches documentaires, des entrevues semi-dirigées et des groupes de discussion. 


\section{3 Étape 3 : Observer}

Dans un premier temps, la collecte des données a été effectuée par des recherches documentaires approfondies sur Internet qui comportaient des articles scientifiques, des rapports, des livres blancs, etc. Cette recension a permis de dresser un portrait descriptif des pratiques d'accompagnement au numérique. La collecte des données a été complétée par des entrevues individuelles et de groupes. En plus de confirmer les pratiques d'accompagnement précédemment identifiées, ces rencontres ont également permis de mieux comprendre comment se déroulent les différents types d'accompagnement recensés, incluant leur conception, leur utilisation ainsi que leur application par les intervenants socioéconomiques concernés. À cet effet, deux intervenants interviewés individuellement évoluaient dans des régions différentes de la province de Québec (Montréal, Mauricie), ainsi que dans deux environnements socioéconomiques différents (urbain, ville de région de moins de 50000 habitants).

Concernant les entrevues de groupes, elles ont été réalisées en Mauricie auprès de deux groupes. Le premier groupe était formé d'entrepreneurs et de dirigeants de PME, tandis que le second groupe était formé d'intervenants socioéconomiques. Ces deux séances ont été l'occasion de comprendre plus précisément les différentes pratiques actuelles d'accompagnement au numérique, incluant les outils de formation et d'évaluation utilisés dans ce contexte (Kushner, 2017). À cet égard, le groupe composé d'intervenants a manifestement contribué à enrichir la collecte des données primaires, notamment concernant leurs perceptions quant au soutien actuellement offert aux entrepreneurs en matière de transformation numérique.

\section{$2.4 \quad$ Étapes 4 et 5 : Analyser et juger}

Bien que l'objectif de la recherche soit initialement descriptif et qu'il consiste d'abord à identifier les pratiques d'accompagnement au numérique, l'équipe de recherche a aussi procédé à une analyse qui a permis non seulement d'identifier les types de compétences soutenues par les différents types d'accompagnement, mais également de déceler quelques impacts anticipés pour chacune des pratiques. L'interprétation des résultats a été faite en tenant compte des éléments identifiés lors de la revue sommaire des écrits scientifiques concernant les compétences numériques. Plus précisément, par un processus interprétatif (Maxwell, 2012), nous avons établi si les types d'accompagnement recensés répondaient aux divers types de compétences numériques que les entrepreneurs et dirigeants de PME devraient acquérir et développer pour optimiser les effets de l'adoption et de l'utilisation des TI dans leur organisation, et ce, selon la catégorisation de l'OCDE (2016), soit les compétences numériques spécialisées, complémentaires ou génériques.

Par la même occasion, différents impacts ont également pu être identifiés concernant l'innovation potentielle découlant de l'accompagnement réalisé. À cet effet, un tableau a été dressé afin d'obtenir le portrait global de la situation ${ }^{3}$. Ce dernier regroupe et structure les données recueillies, soit: les pratiques d'accompagnement, leurs descriptions, les types de compétences numériques ciblées, les impacts anticipés sur le plan organisationnel, ainsi que des exemples permettant de mieux voir à quel(s) type(s) d'intervention(s) l'accompagnement se destinait, incluant les clientèles visées et les objectifs des programmes offerts.

\section{5 Étape 6 : Diffuser}

Toujours en phase de diffusion par l'intermédiaire de différentes activités passées et à venir (colloque de l'ACFAS, rapport de recherche, articles scientifiques, conférences destinées à des auditoires variés, etc.), les résultats de la recherche ont contribué à enrichir les connaissances, à encourager l'évaluation de l'accompagnement réalisé, à faciliter les prises de décisions concernant les programmes offerts, ainsi qu'à soutenir les échanges entre les partenaires et acteurs de la transformation numérique des PME, et ce, telle qu'elle se réalise désormais dans le contexte actuel des affaires.

\section{Résultats et discussion}

Le tableau 1 présente l'offre actuelle de programmes et de mesures destinés à encourager la transformation numérique des PME de même que l'acquisition et le développement de compétences numériques. Cette offre comporte 19 types d'accompagnement provenant de différentes sources, selon des formules variées. 
1. Agences spécialisées

2. Cabinets-conseils et de consultation

3. Cellules

4. Centres d'expertise

5. Coaching

6. Comités sectoriels et groupes syndicaux

7. Concours

8. Conférences et salons

9. Espaces de travail à partager (coworking)

10. Établissements scolaires
11. Événements de réseautage

12. Fablabs et bacs à sable

13. Incubateurs et accélérateurs

14. Laboratoires traditionnels/numériques

15. Mentorat traditionnel/numérique

16. Organismes de type OSBL

17. Programmes gouvernementaux/municipaux et tables régionales

18. Réseaux

19. Sites web/ressources en ligne

Tableau 1 - Types d'accompagnement au numérique destinés aux PME

Au cours des dernières années, les programmes et mesures d'accompagnement au numérique se sont multipliés aux échelles provinciale et nationale. Les PME de toutes tailles et de secteurs confondus se retrouvent ainsi face à une offre grandissante permettant d'acquérir, d'améliorer et de valider leurs compétences sur le plan non seulement technologique, mais aussi managérial.

En outre, à la lumière de la logique dominante de services présentée par Vargo et Lusch (2017) et leurs collègues (Lusch et Nambisan, 2015; $\mathrm{Ng}$ et Vargo, 2018; Vargo et Akaka, 2012), nous avons établi que le processus d'accompagnement tel qu'il est réalisé actuellement contribue à mieux structurer l'écosystème des services destinés aux PME en général (Pelletier et Cloutier, 2019), incluant le volet numérique, qui est en émergence certaine (Pelletier et Martel, 2019). À cet effet, nous constatons que cet écosystème s'appuie déjà sur certains éléments-clés présents dans le cadre conceptuel proposé afin de favoriser la cocréation de valeur fondée sur les TI, de même que la collaboration entre les différents acteurs concernés (voir figure 1).

De plus, en nous appuyant sur l'analyse précédemment réalisée, nous avons pu mettre en perspective la logique dominante de services et certains impacts particuliers de ces accompagnements, et ce, sur deux plans. Le premier, plus direct, concerne les individus et leur organisation (p. ex., les ressources humaines, technologiques, financières, le savoir/la pratique, la formation du personnel, la gestion du risque, les processus de développement mobilisés ou non). Le second, plus indirect, se manifeste en lien avec l'environnement externe et l'écosystème, notamment par l'intermédiaire d'une collaboration accrue et par le développement d'un meilleur leadership ou d'une stratégie mieux adaptée aux technologies numériques actuellement utilisées par les entreprises et leurs partenaires (Trépanier et collab., 2012). Ainsi, nous constatons qu'en contexte d'accompagnement au numérique, certains éléments relatifs aux dimensions d'INNOSTICMD s'intègrent et enrichissent de manière intéressante la logique dominante de services telle qu'elle est présentée par ses auteurs. Voyons plus précisément comment.

\subsection{Acteurs impliqués (dans...)}

Considérant que l'interaction entre différents acteurs est le fondement même de l'accompagnement des entrepreneurs ou des autres dirigeants de la PME, notamment pour l'acquisition de compétences en TI (Raymond et Uwizeyemungu, 2017), nous pouvons relever deux caractéristiques concernant la logique dominante de services dans ce contexte. La première est que, si ce sont les « accompagnateurs » qui offrent initialement de la valeur par l'intermédiaire de leurs programmes et de leurs mesures d'accompagnement au numérique, c'est « l'accompagné » qui contribue concrètement à la création autant qu'à la capture de cette valeur en l'utilisant pour introduire de l'innovation de nature technologique dans l'organisation (de Vasconcelos Gomes et collab., 2018). La seconde caractéristique est que la cocréation de valeur ne peut avoir lieu selon la perspective de la logique dominante de services que si les acteurs-clés contribuent de manière sérieuse, véritablement collaborative et engagée à cet écosystème de services, existant ou en émergence (Prasad et Green, 2016). 


\subsection{Intégration des ressources (et...)}

Trépanier et ses collègues (2012) indiquent que «pour innover, il faut disposer de diverses ressources : physiques, technologiques, humaines, financières, informationnelles et sociales » (p. 4). En contexte spécifique d'accompagnement au numérique, l'innovation est indubitablement facilitée lorsque les acteurs favorisent la mise en commun des ressources dans l'écosystème de services (de Vasconcelos Gomes et collab., 2018; Lusch et Nambisan, 2015). Ainsi, en distinguant les ressources-clés pouvant affecter le processus d'innovation, les acteurs impliqués et situés au cœur même de l'intégration de ces ressources deviennent non seulement des acteurs économiques, mais aussi sociaux. Ils agissent et interviennent alors à différents titres (p. ex., comme développeurs, intégrateurs technologiques, conseillers stratégiques, mentors ou bailleurs de fonds). Enfin, dans une perspective écosystémique de la transformation numérique, il est également plausible de croire que l'intégration organisationnelle des ressources peut être traitée de manière similaire à un processus d'innovation. Processus où s'établit une communication constante entre les intervenants dans l'écosystème, notamment par l'intermédiaire de la collaboration, par une attitude positive à l'égard du changement ainsi que par la collecte et la diffusion de l'information entre les acteurs (Trépanier et collab., 2012).

\section{3 Échange de services (soutenus et contraints par...)}

Considérant la nature sociale autant qu'économique de l'acquisition de compétences numériques par l'intermédiaire de l'accompagnement au numérique (Pelletier et Martel, 2019), il importe de souligner que les acteurs possèdent un double rôle au sein de cet écosystème. En ce sens, ils agissent à la fois comme sources et bénéficiaires de l'échange de services, plus particulièrement grâce au partage des savoir-faire et des connaissances de part et d'autre. Cet aspect de l'accompagnement au numérique rejoint ainsi l'apprentissage au sens large (Trépanier et collab., 2012). D'autant plus que la richesse de l'écosystème s'affiche à son meilleur lorsque les acteurs s'adaptent et innovent, notamment lors de l'intégration des ressources auxquelles ils ont accès (Neirotti et collab., 2018). Par exemple en partageant leurs connaissances et leurs savoirs avec d'autres systèmes organisationnels et institutionnels qui peuvent contribuer à enrichir les échanges de services actuels et futurs ( $\mathrm{Ng}$ et Vargo, 2018). Dans ces conditions, il est alors possible de constater l'importance de la collaboration avec l'environnement externe pour l'amélioration et le développement de l'accompagnement au numérique (Prasad et Green, 2016), plus particulièrement pour les PME (Pelletier et Cloutier, 2019). Réalisé dans un contexte de partage et d'enrichissement mutuel, un tel accompagnement permet alors de faciliter l'acquisition et le développement des compétences numériques, et ce, tout en stimulant la cocréation de valeur dans l'ensemble de l'écosystème et des acteurs qui s'y trouvent.

\subsection{Institutions et arrangements (générés de manière endogène...)}

Comme l'illustre le tableau 1, l'offre de services en matière de transformation numérique provient de différentes sources, à la fois privées, publiques et parapubliques (Gregory et collab., 2015; OCDE, 2016). Pensons ici à la variété des programmes de formation offerts dans les établissements scolaires, aux subventions gouvernementales concernant des thèmes spécifiques du virage numérique (p. ex., industrie 4.0, affaires électroniques, etc.), aux organismes socioéconomiques et OSBL offrant coaching et financement combinés, ou encore aux agences spécialisées privées où l'on trouve une panoplie de spécialistes des TI et autres professionnels de la transformation numérique. Ainsi, un écosystème de services dont certaines activités seraient dédiées à l'accompagnement au numérique doit non seulement contribuer à la cocréation de valeur en promouvant des programmes et des mesures accessibles dans divers marchés, mais également fournir les ressources adéquates pour l'intégration des ressources et les échanges de services qui suivront entre les acteurs-clés, et ce, dans des conditions parfois spécifiques liées à un lieu déterminé (p. ex., villes et villages en déclin économique, régions en forte croissance, diversification des activités économiques traditionnelles, etc.).

Puisque la transformation numérique et son accompagnement, peu importe leur forme, vont bien au-delà de l'entreprise elle-même, il est aussi pertinent de réfléchir aux réseaux, aux territoires et à la portée géographique relative à l'offre de services 
(Daidj, 2011). Dans ces conditions, c'est l'environnement externe lui-même qui met de l'avant la collaboration et l'accès aux ressources externes pour les PME. Cela souligne l'importance du territoire dans lequel se situent l'entrepreneur, l'entreprise et l'intervenant lors d'un projet de transformation numérique, plus précisément en ce qui concerne le degré de proximité des acteurs, l'intensité et la nature de leurs relations ou encore le degré et l'ouverture de leurs contributions respectives à une plateforme numérique commune, le cas échéant (Tsujimoto et collab., 2018). Autant d'éléments ayant des impacts positifs ou négatifs au succès de l'accompagnement réalisé auprès de PME alors organisées en réseaux territorialisés (Daidj, 2011). À cet égard, pensons ici aux établissements d'enseignement (cégeps et universités) qui développent des créneaux d'expertise, aux centres de recherche spécialisés qui y sont parfois rattachés ou encore à certains organismes et sociétés de financement dont la mission serait régionalisée.

Dans cette perspective, l'offre de services et les types d'accompagnement au numérique varieront donc selon le territoire dans lequel l'entrepreneur et son entreprise se situent. À cet effet, les types d'accompagnement au numérique permettant une plus grande portée géographique seront possiblement plus sollicités, incluant ceux qui brisent la frontière territoriale physique. Par exemple en utilisant les outils du Web, et ce, même si d'autres impacts négatifs peuvent être anticipés si l'accompagnement repose exclusivement sur une solution numérique (Gregory et collab., 2015). Dans ce sens, nous pouvons aussi supposer que les «accompagnateurs » gagneront à échanger davantage sur une base continue leurs savoirs et leurs pratiques entre eux, et ce, en amont de l'accompagnement réalisé afin de véritablement enrichir le travail réalisé en aval auprès de l'entrepreneur ou du dirigeant « accompagné ».

\section{5 Écosystèmes de services (existants, nichés et imbriqués)}

En matière de transformation numérique des PME, il ne suffit pas de mettre en ligne un site web de l'entreprise pour se faire connaître de la planète entière, de même que l'implantation d'un robot dans l'usine ne règlera pas, comme par magie, la pénurie de main-d'œuvre. Ainsi, adopter une perspective écosystémique qui reconnaît la complexité de cette transformation pour les PME contribue assurément à bâtir une vision stratégique commune aux organisations impliquées. Une telle perspective favorise également une meilleure communication aux acteurs-clés concernés (Trépanier et collab., 2012) de même qu'elle stimule la créativité ainsi que l'ouverture aux aspects relationnels du processus d'accompagnement au numérique (Dyer et Kale, 2007). Cette stratégie devra toutefois être axée sur la satisfaction des besoins de l'ensemble des acteurs (clients, utilisateurs, intervenants, fournisseurs, etc.), car, rappelons-le, tous ces individus jouent un double rôle dans le processus de cocréation de valeur. C'est aussi pourquoi il est nécessaire de cultiver une attitude positive à l'égard de l'innovation qui soit fondée sur les TI et les compétences numériques désormais exigées (Pelletier et Cloutier, 2019). Cela tout au long de l'accompagnement réalisé, notamment afin que la mise en commun des idées soit privilégiée dans le reste de l'écosystème (de Vasconcelos Gomes et collab., 2018).

\section{Contributions et conclusion}

Les deux questions posées pour la recherche étaient : Comment se réalise l'accompagnement à la transformation numérique dans un écosystème d'affaires dédié aux entrepreneurs et PME? Qu'estce qui caractérise les relations qu'entretiennent alors les différents acteurs de cette transformation numérique? En réponse à celles-ci, la démarche a permis de nommer 19 types d'accompagnement au numérique actuellement offerts aux PME. Plus précisément et en plus de, leur identification plus précise sur le plan descriptif, l'analyse subséquente contribue aux connaissances en encourageant la distinction et l'évaluation des types d'accompagnement réalisés, et ce, afin de mieux soutenir les acteurs qui y agissent ou interagissent individuellement, en groupes et en réseaux.

Un autre objectif de la recherche était d'éclairer l'aspect social du système complexe dans lequel se trouvent les entrepreneurs et les PME par rapport au passage de plus en plus obligé de la transformation numérique, soit l'accompagnement qui leur est offert par l'intermédiaire de leur écosystème qui inclut d'autres utilisateurs, des intervenants ou des spécialistes. Ce faisant, une meilleure compréhension du rôle des acteurs externes à l'entreprise a été mise de l'avant, de même que le capital social qui se constitue entre eux. 
Sur le plan pratique, ces nouvelles connaissances facilitent les prises de décisions concernant les programmes offerts, de même qu'elles encouragent les échanges entre les partenaires et acteurs de la transformation numérique des PME, et ce, telle qu'elle se réalise désormais dans le contexte actuel des affaires. Par exemple, lorsqu'il est question d'identifier plus précisément les divers acteurs impliqués dans la cocréation de valeur lors de la transformation numérique réalisée, dans l'intégration des ressources et l'échange de services entre eux, dans les institutions mobilisées ou encore dans les arrangements contractuels (Vargo et Akaka, 2012). À cet effet, certains de ces acteurs, notamment les intervenants socioéconomiques et autres organismes dédiés au développement des PME, se sont avérés être aux premières loges afin de poser les jalons d'une offre mieux adaptée, à la fois aux besoins, au contexte et à la réalité de ces entreprises et de leurs dirigeants. Ces jalons prennent généralement la forme de programmes d'accompagnement au numérique où des fonds publics peuvent être mobilisés, parfois de manière importante, d'où l'importance d'attribuer adéquatement les ressources (Gregory et collab., 2015), autant que de coordonner efficacement les activités (Vargo et Lusch, 2017).

En résumé, la logique dominante de services, combinée à des éléments de l'outil-diagnostic INNOSTIC $^{\mathrm{MD}}$, s'est avérée bien adaptée pour mieux comprendre la situation qui se pose actuellement aux entrepreneurs et aux PME en matière d'accompagnement au numérique. Cette perspective enrichie de la (co)création de valeur et de la transformation numérique permet en outre de mieux orienter, voire de (ré) orienter l'élaboration et la mise en œuvre des mesures de soutien appropriées dans ce contexte.

Enfin, conformément aux visées d'une démarche fondée sur l'évaluation (Kushner, 2017), les résultats obtenus permettent aussi d'énoncer quelques recommandations spécifiquement destinées aux acteurs de la transformation numérique œuvrant ou désirant œuvrer dans les écosystèmes d'affaires du Québec et du Canada. En voici la liste ${ }^{4}$ :
- Conception d'un guide d'intervention spécifique en matière d'accompagnement de la transformation numérique pour les PME qui comptent moins de 10 employés;

- Conception, application et évaluation d'une méthode d'accompagnement au numérique structurée, réplicable et mesurable;

- Personnalisation de l'accompagnement au numérique sur les plans organisationnel (taille, secteur, etc.) et individuel (compétences, poste occupé, expérience, etc.);

- Récurrence et maintien des programmes et des mesures dédiés à l'accompagnement au numérique;

- Planification de la continuité des services d'accompagnement au numérique;

- Neutralité de l'intervention et de l'intervenant vis-à-vis de la personne ou de l'entreprise accompagnée.

En conclusion et bien que de nature exploratoire pour le moment, les résultats et la discussion présentés ici contribueront, nous l'espérons, à faire avancer les connaissances sur un sujet qui demeure encore flou, soit l'accompagnement au numérique, dans un domaine actuel où se pointent des défis importants pour les PME, particulièrement celles de plus petite taille dont les dirigeants peinent à bien évaluer la situation autant que les options qui s'offrent à eux. Présentés à l'aide d'une approche écosystémique, ces mêmes résultats permettent en outre à d'autres acteurs de la transformation numérique (consultants spécialisés, intervenants socioéconomiques, organismes de soutien, bailleurs de fonds, etc.) d'approfondir, à leur tour, la réflexion concernant les enjeux liés aux pratiques d'accompagnement au numérique. Cela inclut les compétences spécifiques qu'ils visent à soutenir, de même que les questions qui pourraient être soulevées concernant la pertinence et la validité de leurs pratiques, programmes et mesures, et ce, qu'ils soient financés ou non par les fonds publics. 


\section{NOTES}

1 L'outil de diagnostic et de gestion stratégique INNOSTICMD a été conçu pour les PME manufacturières. Il propose différents engrenages qui agissent de manière à soutenir l'innovation organisationnelle. Il a été développé et est utilisé au Laboratoire de recherche sur la performance des entreprises (LaRePE) de l'Institut de recherche sur les PME, à l'Université du Québec à Trois-Rivières. L'information complète concernant cet outil est disponible au https://oraprdnt.uqtr.uquebec.ca/pls/ public/gscw031?owa_no_site $=781$ \&owa_no_fiche $=51$

2 Pour des exemples précis d'interventions ou de programmes existants à cet effet, voir le rapport issu des travaux de Pelletier et Martel (2019).

3 Le rapport présentant les résultats détaillés de la recherche est disponible pour téléchargement au www.uqtr.ca/Claudia.Pelletier (onglet « Publications et communications »).

4 Pour une explication détaillée des recommandations émises, voir le rapport issu des travaux de Pelletier et Martel (2019).

\section{RÉFÉRENCES}

Barney, J. B., Ketchen, D. J. et Wright, M. (2011). The future of resource-based theory. Journal of Management, 37(5), 1299-1315. https://doi,org/10.1177/0149206310391805

Barthelémy, M.-L. et Le Pennec, H. (2018). La boîte à outils de l’accompagnement professionnel. Malakoff, France : Dunod.

Daidj, N. (2011). Les écosystèmes d'affaires : une nouvelle forme d’organisation en réseau? Management \& Avenir, 6, 105-130. https://doi.org/10.3917/mav.046.0105

de Vasconcelos Gomes, L. A., Facin, A. L. F., Salerno, M. S. et Ikenami, R. K. (2018). Unpacking the innovation ecosystem construct: Evolution, gaps and trends. Technological Forecasting and Social Change, 136, 30-48. https://doi.org/10.1016/j.techfore.2016.11.009

Dyer, J. H. et Kale, P. (2007). Relational capabilities: Drivers and implications. Dans C. E. Helfat (dir.), Dynamic capabilities understanding strategic change in organizations (p. 65-79). Oxford, R.-U. : Blackwell Publishing.

Gregory, R. W., Keil, M., Muntermann, J. et Mähring, M. (2015). Paradoxes and the nature of ambidexterity in IT transformation programs. Information Systems Research, 26(1), 57-80. https://doi.org/10.1287/isre.2014.0554

Gross, N. (2009). A pragmatist theory of social mechanisms. American Sociological Review, 74(3), 358-379. https://doi.org/10.1177/000312240907400302

Innovation, Sciences et Développement économique Canada (2019). Principales statistiques relatives aux petites entreprises : janvier 2019. Ottawa, ON : Gouvernement du Canada. Repéré à www.ic.gc.ca/eic/site/061.nsf/vwapj/PSRPE-KSBS_Jan_2019V2_fra.pdf/\$file/PSRPE-KSBS_Jan_2019-V2_fra.pdf

Kushner, S. (2017). Evaluative research methods: Managing the complexities of judgment in the field. Charlotte, NC : Information Age Publishing.

Laudon, K. C., Laudon, J. et Costa, S. (2017). Management des systèmes d’information (15e éd.). Montreuil, France : Pearson France.

Lusch, R. F. et Nambisan, S. (2015). Service innovation : A service-dominant logic perspective. MIS Quarterly, 39(1), 155-176. https://doi.org/10.25300/MISQ/2015/39.1.07

Maxwell, J. A. (2012). Qualitative research design: An interactive approach (3e éd.). Thousand Oaks, CA: SAGE Publications.

Neirotti, P., Raguseo, E. et Paolucci, E. (2018). How SMEs develop ICT-based capabilities in response to their environment: Past evidence and implications for the uptake of the new ICT paradigm. Journal of Enterprise Information Management, 31(1), 10-37. https://doi.org/10.1108/JEIM-09-2016-0158

Ng, I. C. et Vargo, S. L. (2018). Service-dominant (SD) logic, service ecosystems and institutions: Bridging theory and practice. Journal of Service Management, 29(4), 518-520. https://doi.org/10.1108/JOSM-07-2018-412

Organisation de coopération et de développement économiques (OCDE). (2016). Quelles compétences pour un monde numérique? Paris, France : OCDE. Repéré à www.oecd.org/fr/internet/ministerielle/reunion/Panel-4.2-FR.pdf

Pelletier, C. et Cloutier, L. M. (2019). Challenges of digital transformation in SMEs: Exploration of IT-related perceptions in a service ecosystem. Dans Proceedings of 52nd Hawaii International Conference on System Sciences (HICSS), Grand Wailea, HI.

Repéré à https://scholarspace.manoa.hawaii.edu/bitstream/10125/59934/0494.pdf 
Pelletier, C. et Martel, V. (2019). Pratiques d'accompagnement au numérique : soutien au développement des compétences numériques des petites entreprises (Rapport de recherche). Trois-Rivières, QC : Institut de recherche sur les PME (INRPME), Université du Québec à Trois-Rivières.

Prasad, A. et Green, P. (2016). On information technology competencies for collaborative organizational structures. Communications of the Association for Information Systems, 38, 377-399. https://doi.org/10.17705/1CAIS.03822

Raymond, L. et Uwizeyemungu, S. (2017). Les technologies de l'information et les affaires électroniques : levier de la compétitivité des PME industrielles dans une économie mondialisée. Dans J. Saint-Pierre et F. Labelle (dir.), Les PME : d'bier à demain (p. 233-266). Québec, QC : Presses de l’Université du Québec.

Sibieude, T. et Claverie, C. (2011). La mesure de l'impact social: après le temps des discours, voici venu le temps de l'action. Rapport préparé pour le Conseil supérieur de l'économie sociale et solidaire (ESS). Paris, France : CSESS. Repéré à www.avise.org/sites/default/files/atoms/files/20140204/201112_CSESS_Rapport_ImpactSocial.pdf

Trépanier, M., St-Pierre, J. et Bertrand, J. (2012). «Diagnostiquer » la capacité d’innovation des PME par un outil systémique : INNOSTIC ${ }^{2}$, Bulletin InfoPME, 12(1). Repéré à https://oraprdnt.uqtr.uquebec.ca/pls/public/docs/FWG/GSC/Publication/160/34/1237/1/46081/3/F466307688_InfoPME_Janv_2012.pdf

Tsujimoto, M., Kajikawa, Y., Tomita, J. et Matsumoto, Y. (2018). A review of the ecosystem concept: Towards coherent ecosystem design. Technological Forecasting and Social Change, 136, 49-58. https://doi.org/10.1016/j.techfore.2017.06.032

Vargo, S. L. et Akaka, M. A. (2012). Value cocreation and service systems (re)formation: A service ecosystems view. Service Science, 4(3), 207-217. https://doi.org/10.1287/serv.1120.0019

Vargo, S. L. et Lusch, R. F. (2017). Service-dominant logic 2025. International Journal of Research in Marketing, 34(1), $46-67$. https://doi.org/10.1287/serv.1120.0019

Weiß, P., Zolnowski, A., Warg, M. et Schuster, T. (2018). Service dominant architecture: Conceptualizing the foundation for execution of digital strategies based on SD logic. Dans Proceedings of 51 st Hawaï International Conference on System Sciences (HICSS), Big Island, HI. https://doi.org/10.24251/hicss.2018.204

Zollo, M., Bettinazzi, E. L., Neumann, K. et Snoeren, P. (2016). Toward a comprehensive model of organizational evolution: Dynamic capabilities for innovation and adaptation of the enterprise model. Global Strategy Journal, 6(3), 225-244. https://doi.org/10.1002/gsj.1122 